\title{
A New Color on Discrimination Based on Characteristics of the Vehicle License Plate Location
}

\author{
Xiaole Liu ${ }^{\mathrm{a}}$, Cailing Wang ${ }^{\mathrm{b}}$ \\ ${ }^{a}$ Department of Computer Science and Engineering, Henan Institute of Engineering, Zhengzhou 451191, \\ China \\ ${ }^{b}$ Department of Information Security, Henan Police Academy, Zhengzhou 450052, China
}

\begin{abstract}
The license plate recognition system, the license plate location is one of the important technologies, can accurately complete the orientation will directly affect the follow-up of the character segmentation and recognition. This paper presents a new color on discrimination based on characteristics of the vehicle license plate location, Firstly, edge detection, image enhancement preprocessing, effectively highlighting the license plate features, And then by line scanning of the plate rough location, and color-based features to distinguish the license plate location method further extract the real license plate. Experiments show that with the car in a complex background, this method can accurately locate the license plate.
\end{abstract}

Index Terms: License Plate Location; Edge Detection; Line Scanning; Feature Color On

(C) 2012 Published by MECS Publisher. Selection and/or peer review under responsibility of the International Conference on E-Business System and Education Technology

\section{Introduction}

Recently, with the rapid development of highway and the wide use of vehicle, people begin to pay more and more attention to the intelligent transportation system(ITS). The vehicle license plate recognition is one of the important researches in the field of computer vision or digital image processing, which widely used in the intelligent transportation system. The vehicle license plate recognition is used for detecting speeding cars, security control in restricted areas, unattended park zone, traffic law enforcement and electronic toll collection.

There are three steps in the vehicle license plate recognition. That is vehicle license plate location, character segmentation, and character recognition. The task of the vehicle plate location is to find license plate area from vehicle image, which is the key step in the vehicle license plate recognition. Whether it can locate the accurate position of the license plate has great effect on the performance of the vehicle license plate recognition.

Corresponding author:

E-mail address: ${ }^{\text {aWo9shi9@163.com ; b65376854@qq.com }}$ 
The focus of this paper is on a new vehicle license plate location method which is based on the combination of line scan and feature color. The experiment results show that the proposed method is very effective in coping with different conditions such as pool illumination, varied distance from the vehicle and varied whether.

\section{Preprocessing of License Plate Image}

The license plate images usually have high-resolution and the image will be different degrees of light and other interference, especially under the license plate image in a complex environment may be blurred, or even deformation of the follow-up treatment is very negative. The image preprocessing will give prominence to the information of object, and weaken unnecessary information and interference noises.

\subsection{Edge detection}

Edge detection is either based on gray image or color image. The drawback of gray image-based edge detection is that under strong light environment it will bring more edge and wide fringe. And there is no such problem in the color image-based edge detection. Zhang etc. in [1] proposed the ColorPrewitt Operator and ColorPrewitt Operator.

Because the change of color and the brightness of the license plate image in more violent in the horizontal direction is more violent than that of in the vertical, we use the ColorPrewitt operator to only be obtained using color edge operator gradient image levels, to better highlight the license plate area In this paper, we use ColorPrewitt operator to extract to the edge of license plate image.

The method of the ColorPrewitt operator is as follow

$$
\begin{aligned}
P_{c} & =e(i-1, j-1 ; i-1, j+1)+e(i, j-1 ; i, j+1) \\
& +e(i+1, j-1 ; i+1, j+1)
\end{aligned}
$$

here,

$$
e\left(i_{1}, j_{1} ; i_{2}, j_{2}\right)=\left\{\sum_{k=1}^{3}\left[C\left(i_{1}, j_{1}, k\right)-C\left(i_{2}, j_{2}, k\right)\right]^{1 / 2}\right\}^{1 / 2}
$$

$C(i, j, k)$ is the pixel $(i, j)$ of the image RGB color component, where $k=1,2,3$

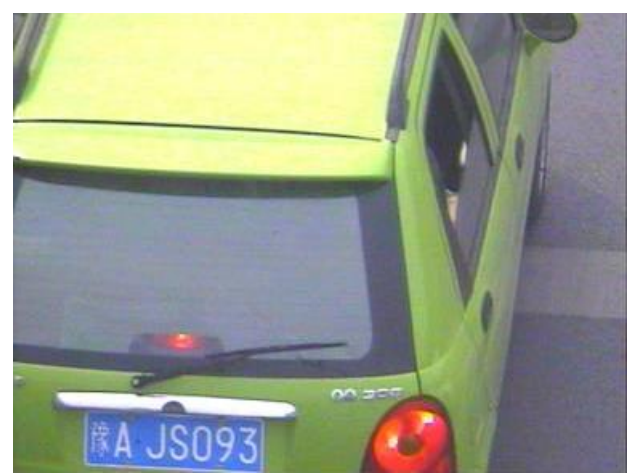

Fig. 1. The original image 


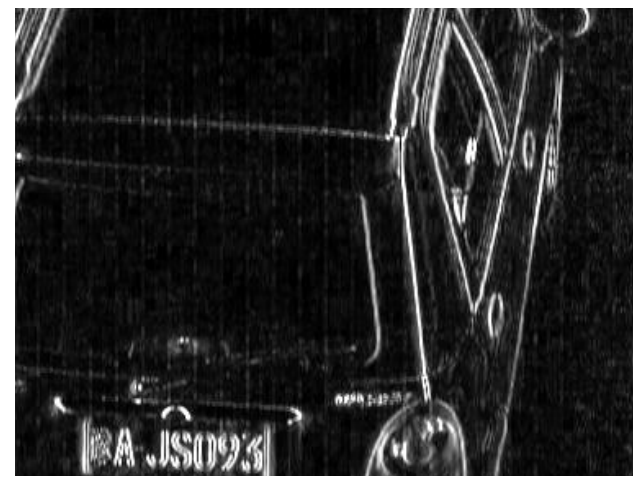

Fig. 2. The image after color Prewitt Operator procession

From the above two image, we can see the plate area is integer and has been enhanced can be easily separate from the background.

In order to avoid the square root operation, we improve the Prewitt operator using the following formula.

$$
e\left(i_{1}, j_{1} ; i_{2}, j_{2}\right)=\sum_{k=1}^{3}\left|C\left(i_{1}, j_{1}, k\right)-C\left(i_{2}, j_{2}, k\right)\right|
$$

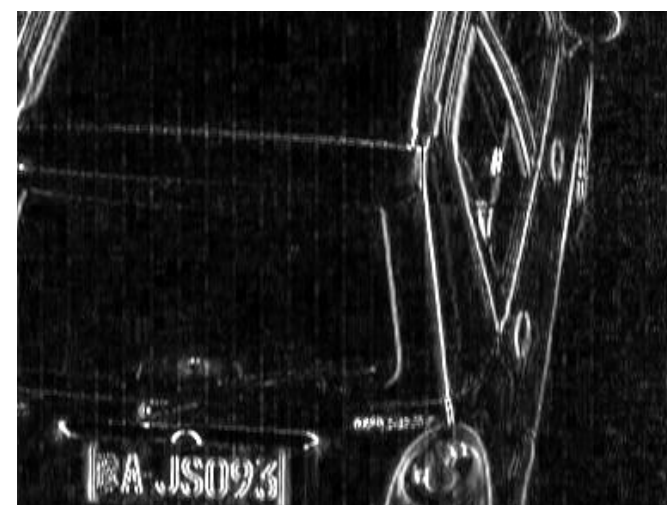

Fig. 3. The Image after improved Prewitt Operator procession

Figure 3 is the original processed by the improved Prewitt Operator. We can be that the improved Prewitt still has good treatment effect. And as there is no square root operation, the processing delay will be greatly reduced.

\subsection{Contrast enhancement}

The images of the license plate images shot under different illumination conditions. As the contrast of image is deficient, which make the character of license plate can not be distinguished and positioned; the effective method must be adopted to enhance the contrast of image. Here, we use gray expanding and Histogram equalization to enhance the contrast of image. 


\section{Gray expanding.}

Gray scale [2] is an effective way to enhance the image contrast. And the gray scale will not change the pixel and only change the gray level of pixel.

Suppose the gray range of the image is $a$ to $b$. Obviously, it does not full use the maximum range of gray range, namely, 0 to 255 . This will make the image contrast too low and some of the details can not be easily observed. Here, we use the follow formula to expand the image scale.

$$
g(x, y)=\frac{255}{b-a}[f(x, y)-a]
$$

Here, $f(x, y)$ is the gray level of the original image on pong $(x, y), g(x, y)$ is the that of the output image.

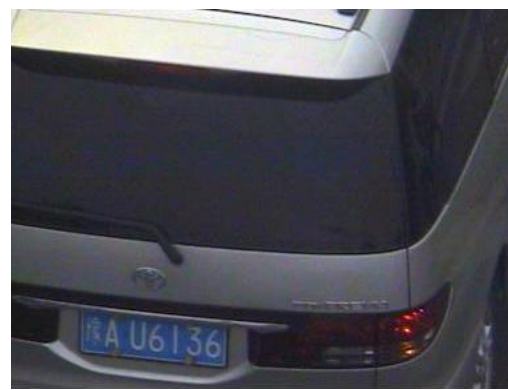

Fig. 4. The original image

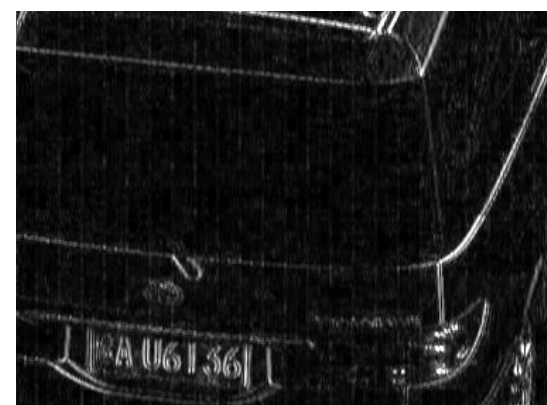

Fig. 5. The image Prewitt Operator procession

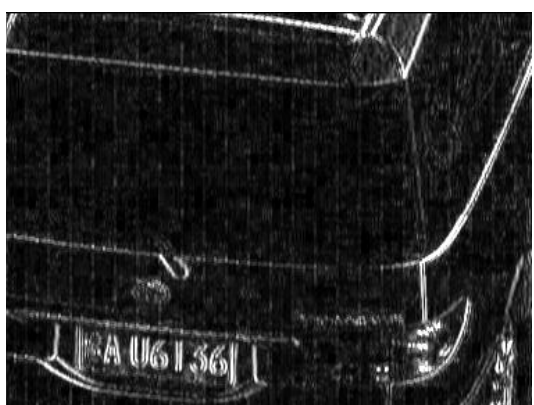

Fig. 6. The Image after procession 
From the above three figures, we can see that gray expanding can effectively enhance the contrast and decrease the averse effects caused by the uneven intensity of light.

\section{Histogram equalization.}

For some license plate image, license plate region and its adjacent areas are dark; the gray expanding will not have much affect on the contrast of image. In this case, we need use image histogram equalization to enhance image contrast.

Histogram equalization [4] is to compress the part in which there are few pixels and stretch the part in which there are many pixels [4]. The gray level of the license plate area usually concentrate on a small range, so the histogram equalization can make the whole image become clearer and the contrast be enhanced.

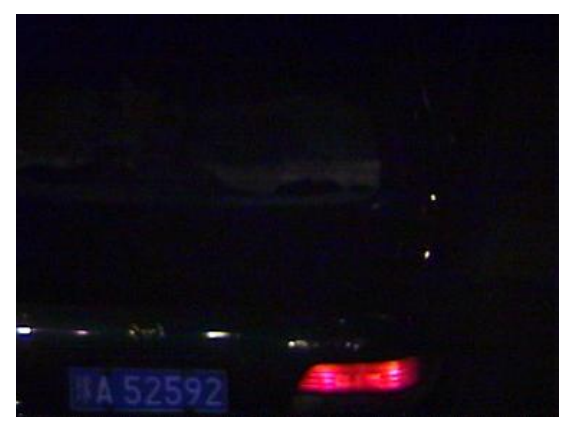

Fig. 7. The original image

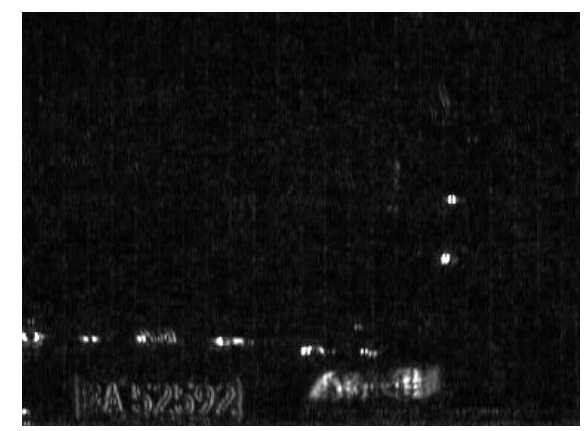

Fig. 8. The image after Prewitt Operator procession

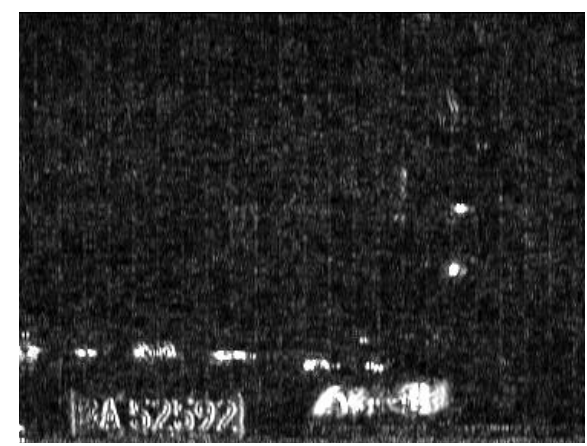

Fig. 9. The image after histogram equalization procession 


\subsection{Binaryzation}

We will get a gray image after the process of the gray expanding and Histogram equalization. In order to facilitate further extraction of license plate, the gray images need to translate to binary image. Binaryzation is the process to map the image gray value to 0 or 1 . That is there are only black and white points in the image [5]. In order to obtain the ideal binary image, we use the method the whole threshold segmentation.

In the whole threshold segmentation, it will select a fixed threshold, which bases on the spatial distribution of gray scale images, to translate the gray image to binary image.

Suppose there is a $M \times N$ image, $f(x, y)((0 \leq x<M, 0 \leq y<N))$ is the gray value of point $(x, y)$. The binaryzation process can be finished by following formula.

$$
f(x, y)= \begin{cases}1, & f(x, y) \geq T \\ 0, & \text { else }\end{cases}
$$

Here, $T$ is the threshold, which is compute by Otsu algorithm proposed in[6].

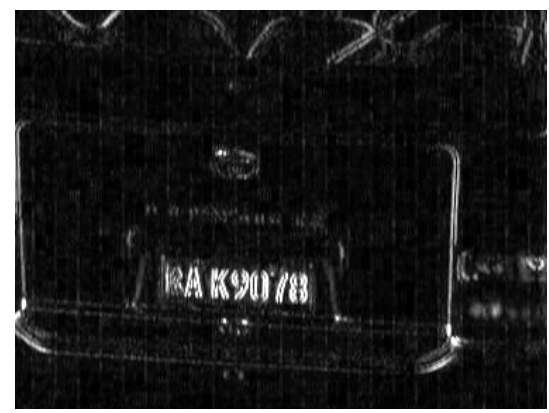

Fig. 10. The Image after Prewitt Operator procession

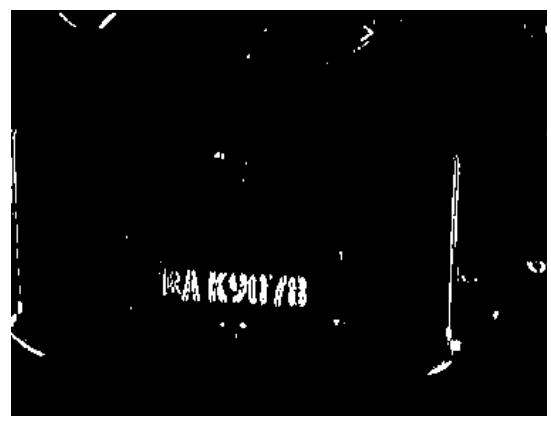

Fig. 11. The image after binaryzation

\subsection{Median filter}

In order to further improve the accuracy of license plate location, the image is need to progressed using the median filter to remove noise while preserver the edge of plate area. 
The main idea of the median filter is replace each point value with the median of neighboring point value s. The pattern of neighbors is called the "window", which slides, point by point, over the entire image. For 1D signals, the most obvious window is just the first few preceding and following entries, whereas for 2D (or higher-dimensional) signals such as images, more complex window patterns are possible (such as "box" or "cross" patterns). If the window has an odd number of entries, then the median is simple to define: it is just the middle value after all the entries in the window are sorted numerically.

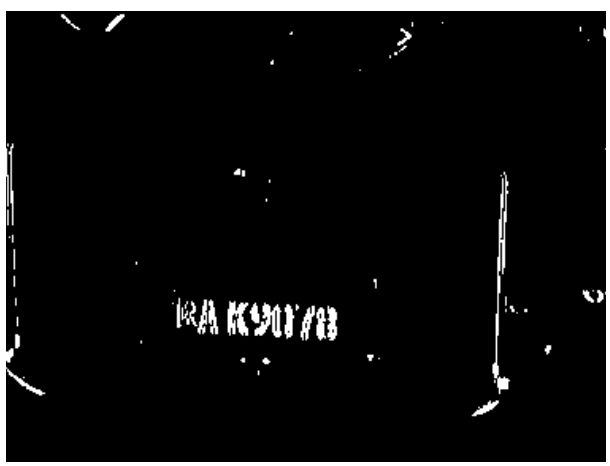

Fig. 12. The image after binaryzation

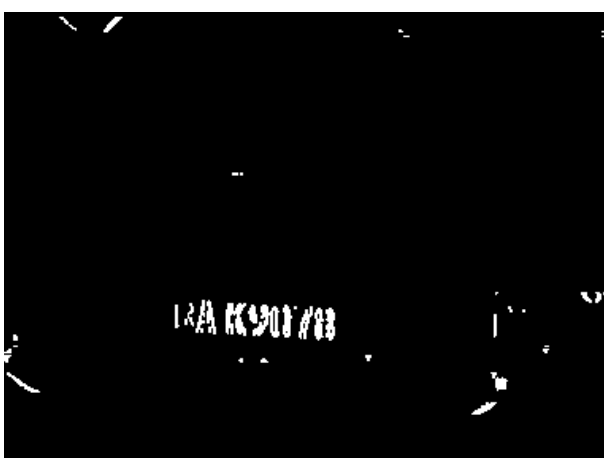

Fig. 13. The Image after median filter procession

\section{Lincese Plate Location}

\subsection{Line sanning-based method.}

After preprocessing, the locating the position of license plate area is the key step to identity the plate number. Considering the real time and robust of the algorithm, we first use line canning-based method to obtain license plate candidate regions.

The basic idea line scanning-based method is simple. We first scan the image by line and row by row from top to bottom, bottom, left to right, right to left. During the scan processing from top to bottom and from bottom to top, if the number of the white pixels is greater than 16, we record the line location in the row when the first encounter white pixel which the number of white pixels is greater than 16 in a column. And so on, when scanning from left to right, from right to left, we record the line location in the column when the first 
encounter white pixel which the number of white pixel is greater than 10 in a row. After getting these four values, we need to do some adjustment based on the actual images.

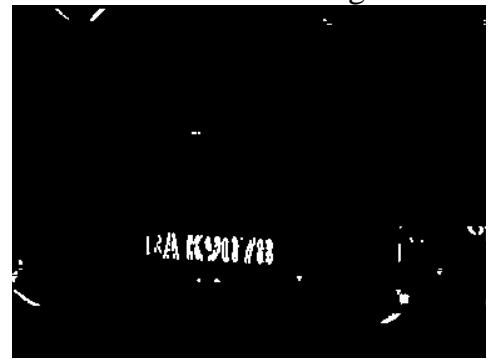

Fig. 14. Schematic diagram of license plate location

There is much interference in the actual environment, especially in the more complex environment to get the license plate image. In order to improve the positioning accuracy, we will do three times to get three plate candidate regions.

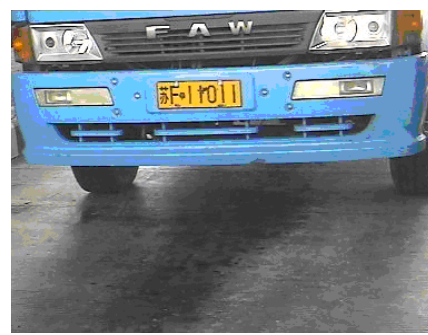

Fig. 15. The original image

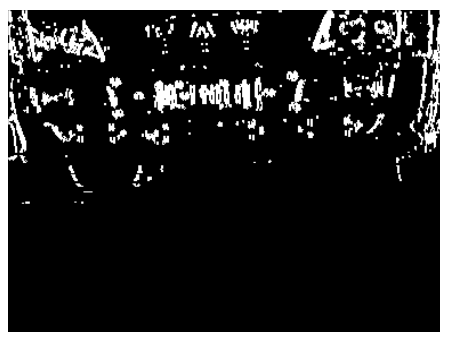

Fig. 16. The Image after pre-procession

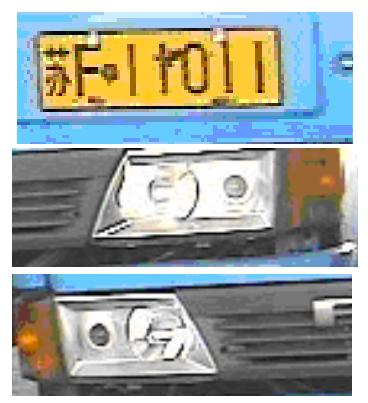

Fig. 17. Coarse positioning plate results 


\subsection{Feathres color-based method.}

After getting the three candidate area, we need to decide how to extract the real license plate region from these candidates. There are three sorts of color combination of front ground and background of license plate in China, such as blue-white, yellow-black, and white-black. Therefore the number of color pair transitions is three. If we find there are many the same color pair transitions, we will consider that this candidate is the real license plate area, and can determine the color the license plate.

As there may be errors in line scanning-based method, the number of the same color pair transitions in the candidate regions almost equal. In this case, the selected license plate area may be wrong. In the real situation, the center of the selected area is sure to be in the license plate area. According to this consideration, we can determine the real license plate area according to the number of the same color pair transition in the center of the pre-selected license plate area. The blow figure demonstrates this method. The largest region is the license plate pre-determine region. The inner frame of the pre-determine region is the license plate real position. The three lines is the statistical area, and these three lines is the centers of the license plate area extend 20 pixels to left, right, top and bottom.

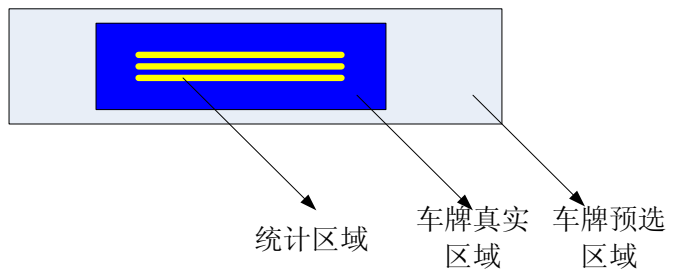

Fig. 18. Color diagram of the statistical area

The identification process as follows:

First, we get the number of the three color pair transition in the three pre-determined area.

Second, we compare the number of the blue-white, yellow-black, and white-black transition in each predetermined area, and record the maximum value.

Third, we compare the maximum of the color-pair transition in the three candidate area. The license plate real area is the candidate area in which the maximum of the color pair transition is the greatest in the three candidate area.

Last, based on the results of license identification, we can judge the color of the license plate. For example, if the number of blue-white color pair is the largest, the color of the license plate is blue.

The blow figure is the real license plate area using the above method.
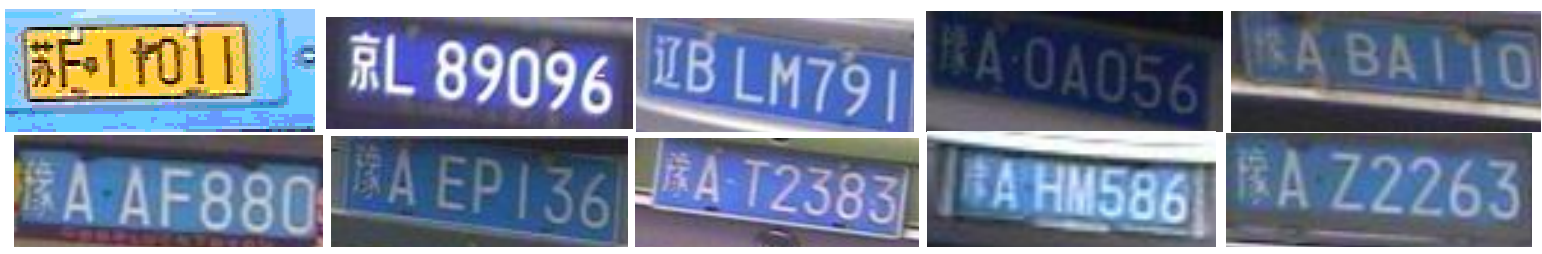

Fig. 19. Actual results of license plate extraction

We use experiment to verify the feasibility and accuracy of this method. The experiment results show that when locating the license plate from 100 license plate images the accuracy rate is $99 \%$. 


\section{Algorithm and Exparimet Results}

According to the above method, we designed the flowchart the license plate location.

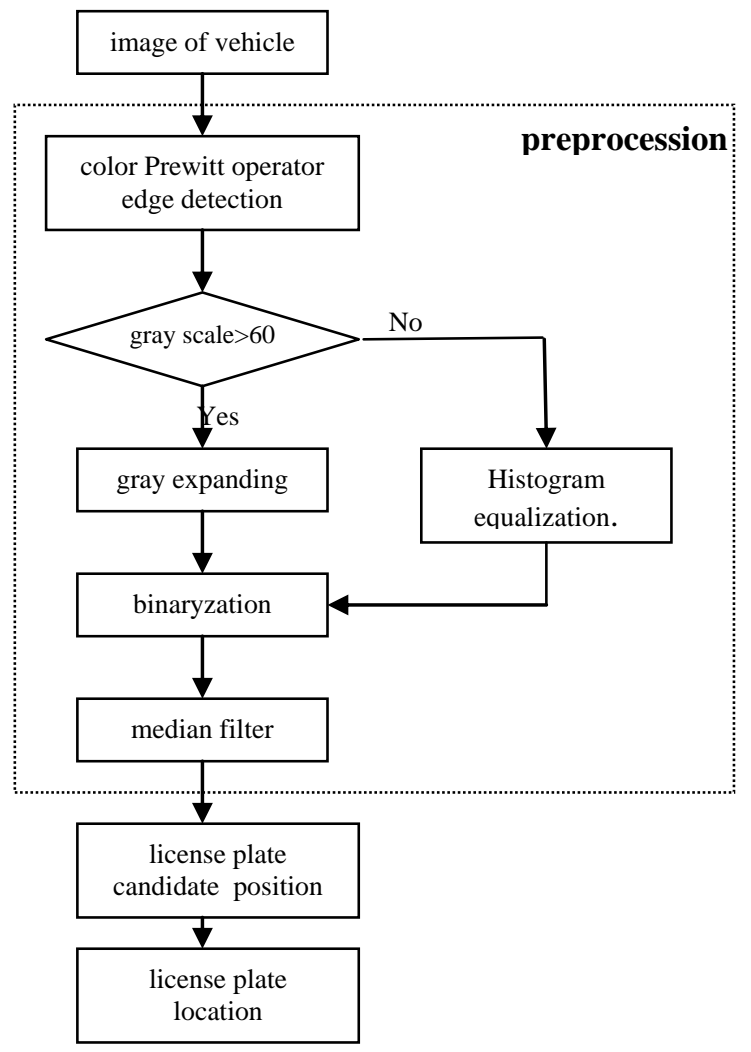

Fig. 20. License plate location flowchart

According the above flowchart, we compare the proposed method, the method based on mathematical morphology and the method based on projection. All experiments were done on Intel (R) Core (TM) 2Duo CPU T72502.00GHz with 2.00 RAM. In the experiments, 100 images were employed. For these images, all of them were taken from key point of road and under different lighting conditions of the real word.

Table 1. Experimental Result License Plate Location Algorithms

\begin{tabular}{lccc}
\hline & The propsed method & Method based on Mathematical Morphology & Method based on projetion \\
\hline Location Accuracy & $99 \%$ & $95 \%$ & $90 \%$ \\
Location Speed & $8 \mathrm{~ms}$ & $14 \mathrm{~ms}$ & $4 \mathrm{~ms}$ \\
\hline
\end{tabular}

From the Table I, we can see that the proposed method is better than the other two methods in location accuracy, and the location speed is faster then the method based on Mathematical Morphology. 


\section{Conclusion}

This paper presents a new vehicle license plate location method based on characteristics of color. This method can highlight the features of license plate and get the vehicle license plate real area. Experiments show that the method of the speed of the positioning of $8 \mathrm{~ms}$, the correct rate was $99 \%$ in the overall positioning rate has been greatly improved. Experiments show that this method can accurately locate the license plate rapidly.

\section{References}

[1] Z. Yin, P. Yunhe, “A new approach for vehicle license plate locating from color image,” Journal of Image and Graphics, vol.6, 2001

[2] J.Xiaojun, Z.Xianwei, and F.Yali. "Image processing and its application", National Defence Industry Press, Beijing, 2005.

[3] Y.Qiuqi. "Digital image processing”, Electronic Industry Press, Beijing, 2001

[4] L.Cunping, and W.Qiang. "Digital image processing and analysis", Qinghua University Press, Beijing, 2006.

[5] J.Xiangang, "Digital image pattern recognition engineering software design", Chian WaterPower Press, Beijing, 2008.

[6] J.Bernsen, "Dynamic thresholding of gray level images", in: Proceedings of 8th International Conference on Pattern Recognition. Paris, France, 1986, pp:1251-1255

[7] Feng Yang, Zheng Ma and Mei Xie. A Novel Approach for License Plate Charater Segmentation[J]. Industronics and Application, 2006 IEEE Coference on May 2006

[8] Takashi Naito,Toshihiko Tsukada,Keiichi Yamada,et,al. Robust license-plate recognition methods for passing vehicles under outside environment[J]. IEEE Transactions on Vehicular Technology, 2000, 49(6):2309-2319

[9] $\mathrm{Li} \mathrm{C}$,Ding $\mathrm{X}, \mathrm{Wu}$ Y.Automatic text location in natural scene images[A] .IEEE Sixth International Conference on Document Analysis and Recognition[C] ,2001,1069-1073

[10] Da Rocha Gesualdi A,De Albuquerque M P.MARCEL ( ) PORTES DE ALBUQUERQUE.Recognition of characters in plates of privale Brazilian vehicles using neural networks[J].IEEE,2000

[11] Sun Guangmin,Li Gang,Xu Lei,eta.A new method of vehicle license plate location based on mathematicalmorphology and texture characteristics[C].Industrial Electronics and Applications.IEEE Conference on 2008:985-988

[12] Vendors-Viotation Enforcement Lisence Plate Recognition.http://www.ettm.corn/vendors_yes.html,2000

[13] Ter M H,Brugge,Stevens J h,Nijhuis J A G.License Plate Recognition Using DTCNNs.In:Proceeding of the IEEE International Workshop on Cellular Neural NetWorks and their Application,1998.212 217 